\title{
A Design of Remote-Controlled Chalkboard Eraser
}

\author{
Haohua Chen, Qidong Zhou \\ Cambridge A-Level Center, Hangzhou Foreign Languages School, Hangzhou, China
}

Correspondence to: Haohua Chen, haoh.chn@gmail.com; Qidong Zhou, 2721589566@qq.com

Keywords: Remote-Control, Arduino UNO Board, Passive Infrared Radiation Sensor, Microfiber, Vacuum Pump

Received: September 24, $2020 \quad$ Accepted: November 10, $2020 \quad$ Published: November 13, 2020

Copyright $\odot 2020$ by author(s) and Scientific Research Publishing Inc.

This work is licensed under the Creative Commons Attribution International License (CC BY 4.0).

http://creativecommons.org/licenses/by/4.0/

\section{(c) (i) Open Access}

\section{ABSTRACT}

Chalkboard erasers that are commonly used in many schools and education organizations have the poor ability in removing the dust off the chalkboard and collect them. The dust that is not collected will be taken into human bodies via inhalation, which will lead to respiratory diseases. Therefore, it is crucial to design a chalkboard eraser that can collect the dust effectively. If the eraser can be controlled both remotely and manually by people, it can further reduce the amount of dust taken in by human bodies. To achieve remote control, a micro-controller is needed to transfer the infrared radiation (IR) into signals that can control electric motors to move around on the chalkboard. Furthermore, the microfiber is also used as the cleaning material to improve the performance of dust cleaning and collecting. A vacuum pump is needed to create negative pressure between the eraser and the chalkboard so that the eraser can stick to it. The result shows a stronger ability in removing and collecting dust. With the help of the Arduino UNO board, the remote control is successfully achieved, and the eraser can move on the chalkboard freely according to the order.

\section{INTRODUCTION}

Long time exposure to chalk dust is common in teachers. According to our observations, although many schools in China, especially in big cities, currently switch to use the whiteboard, electronic blackboard, and dustless chalks to replace the traditional blackboard, using chalks and blackboard is still very common in many schools due to high costs of replacements. The report (Assessment of Airborne Fine Particulate Matter and Particle Size Distribution in Settled Chalk Dust during Writing and Dusting Exercises in a Classroom, 2011) states: "During teaching, entry of chalk dust in the respiratory system through nasopharyngeal region and mouth could be extensive in teachers due to their proximity to the board and frequent opening of mouth during lectures and occasional gasping and heavier breathing due to exhaustion" [1]. As per the current state of knowledge on particulate matter vis-a-vis chalk dust, it "may remain suspended in the air for some time before settling on the floor and body parts of the teachers and pupils". In the short run, the amount of dust inhaled by teachers or students might be tiny, but it can cause respi- 
ratory disease after decades of exposure to this environment. As a result, this study aims to invent a new chalkboard eraser to reduce the risk of chalk dust exposure.

Recent researches have been conducted in reducing the amount of chalk powder produced during the teaching procedure. Most of them focused on improving the design for the chalkboard eraser to reduce the amount of powder produced. One of the main types of design is the manual chalkboard eraser with an electric motor that rotates a fan to take the powder into the eraser [2]. Another type of design is the manual chalkboard eraser without electric motors and fully depends on the mechanical structure to take into the powder [3]. Both types of design achieve satisfactory results in reducing the amount of powder produce; however, neither of them is popularized on the market.

Consider the fact that limited researches have been done about erasers that can clean the chalkboard with the remote-control technology. Meanwhile, we want this eraser to possess satisfactory performance on reducing the amount of chalk powder produced. Therefore, we want to design an electric eraser of this type that saves time for users to clean the chalkboard.

Following the introduction, Materials and methods will describe several functions of the blackboard eraser, including remote-control technology implemented on the Arduino microcontroller, circuit design, the pump system used to create negative pressure, the cleaning material, and the wheel system that carries the eraser around on the chalkboard. Results and discussions will include our research results and future expectations.

\section{MATERIALS AND METHODS}

Our design of the chalkboard eraser is shown in Figure 1, Figure 2 and Figure 3 (unit: $\mathrm{mm}$ ).

The average size of a person's hand ranges from 7.6 to 6.8 inches (193 and $173 \mathrm{~mm}$ ) in length and 3.5 to 3.1 inches $(89$ and $79 \mathrm{~mm}$ ) in breadth respectively [4]. Therefore, we designed the chalkboard eraser 80 $\mathrm{mm}$ wide, $130 \mathrm{~mm}$ long, and $65 \mathrm{~mm}$ thick (including brushes) so that it will be suitable to be held in hand by the majority of people. When the button is pressed downwards, the pump will take out the air inside the sealed chamber. The outer shell will be made if plastic, which is both economic and compact. Details of the remote-control system, the circuit, the pump, the cleaning material, and the wheel system will be illustrated in Sections 2.1, 2.2, 2.3, 2.4, and 2.5 respectively.

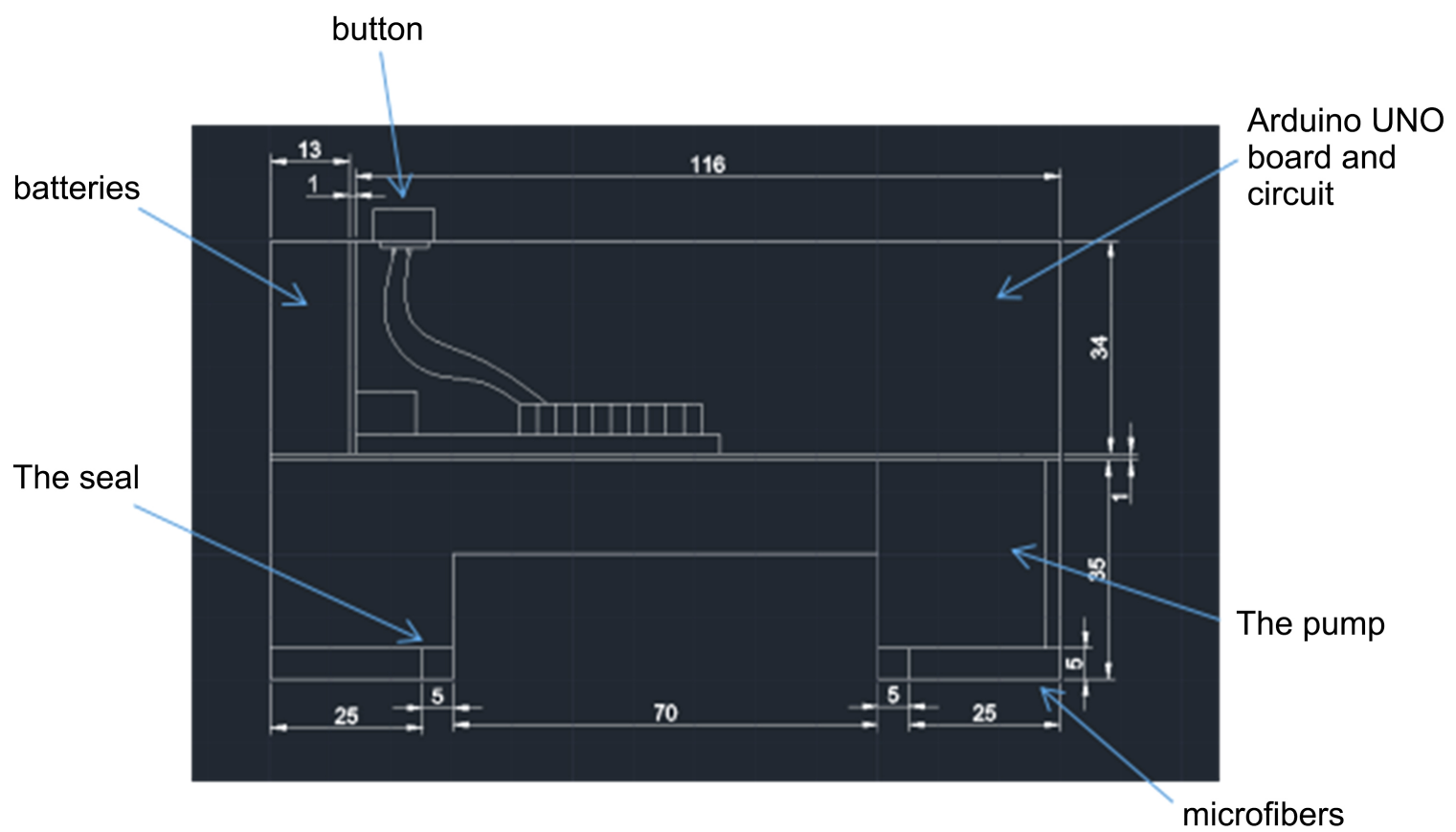

Figure 1. The front view. 


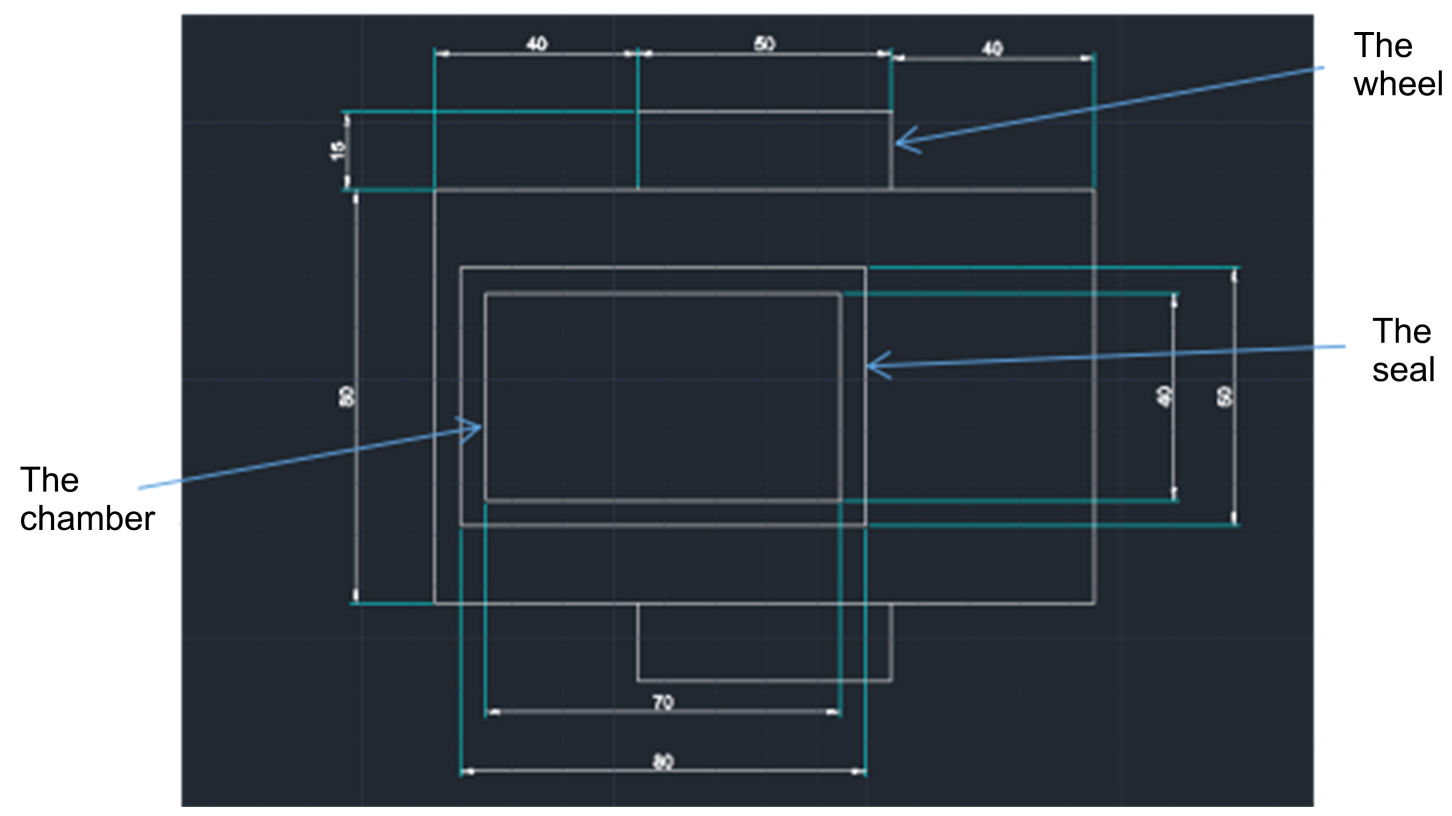

Figure 2. The bottom view.

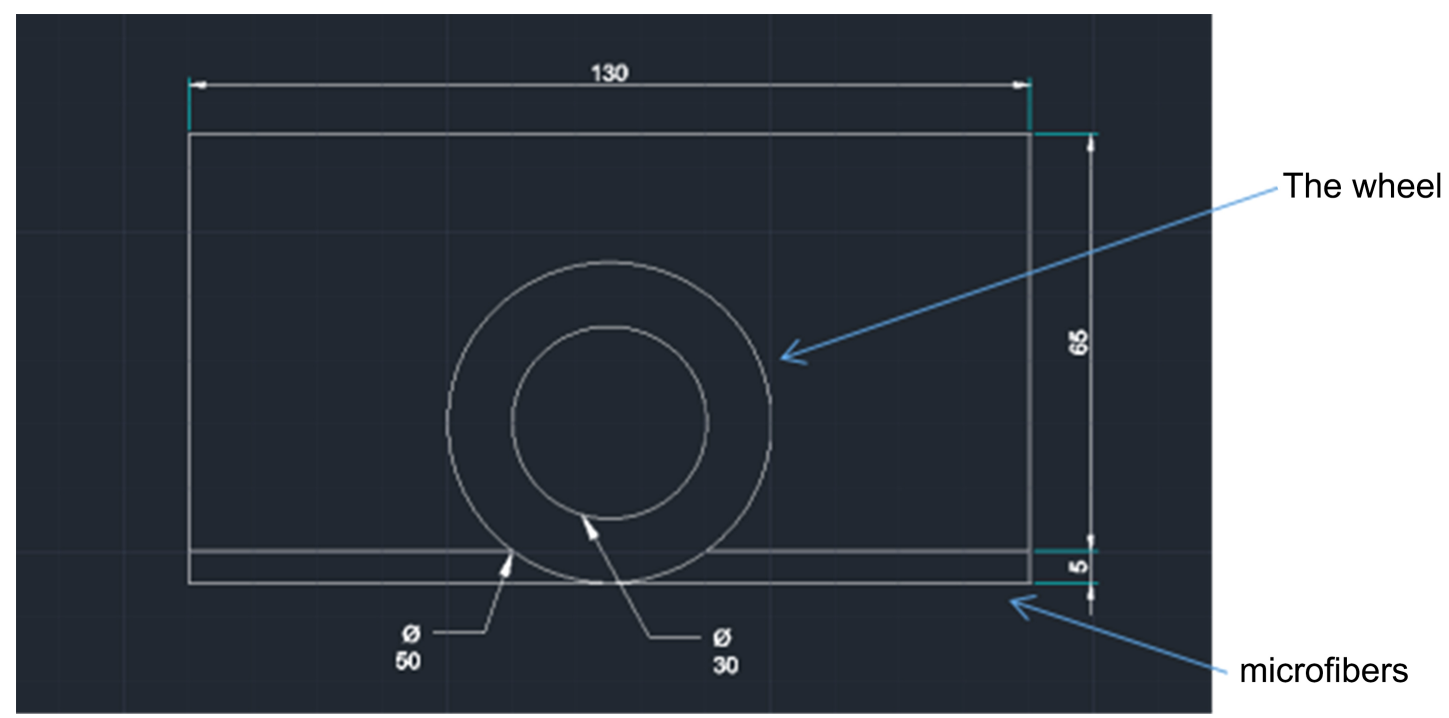

Figure 3. The left view.

\subsection{Remote-Control System}

This paragraph talks about the remote-control system with its design of the eraser. We are inspired by some domestic appliances to design this system. For example, the dominant remote-control technology in home-theater applications which uses the infrared radiation (IR) control.

Infrared radiation was accidentally discovered by an astronomer named William Herchel in 1800. While measuring the temperature of each color of the light (separated by a prism), he noticed that the temperature just beyond the red light was highest. IR is invisible to the human eye, as its wavelength is longer than that of visible light. Infrared light is in the invisible portion of the electromagnetic spectrum and has its wavelength range from $760 \mathrm{~nm}$ to $1 \mathrm{~mm}$ (between the visible light and the microwave of electromagnetic spectrum). Anything that emits heat (everything that has a temperature above around five degrees Kelvin) gives off infrared radiation [5]. 
The basic premise at work in an IR remote control is the use of light to carry signals between the remote control and the device it's directing. An IR remote control (the transmitter) sends out pulses of infrared light that represent specific binary codes. These binary codes correspond to commands, such as Power On/Off and Volume Up. The IR receiver in the TV, stereo, or other device decodes the pulses of light into the binary data (ones and zeroes) that the device's microprocessor can understand. The microprocessor then carries out the corresponding command. An IR sensor is an electronic device that measures and detects infrared radiation in its surrounding environment [6].

There are two main categories of IR sensors currently, one is the passive IR sensor (PIR) and the other is the active IR sensor. Active Infrared Sensor contains both transmitter and receiver. Most of the cases LED or laser diode is used as a source. LEDs for non-imaging IR sensors and laser diode for imaging IR sensors are used. Active IR Sensor works by radiating energy, received and detected by the detector, and further processed by the signal processor in order to fetch information required. It is usually applied in Break Beam Sensor, Reflectance Sensor. For passive IR sensors, it only contains detectors. There won't be a transmitter component. These types of sensors use objects as an IR source/transmitter. The object radiates energy and it is detected by IR receivers. A Signal processor is then used to interpret the signal to fetch information required. It is often used in Thermocouple-Thermopile, Bolometer, Pyro-Electric Detector, etc. In this project, we use PIR in our eraser instead of the active IR because PIR sensors are excellent for detecting general movement [7].

We choose the Arduino UNO board to work as our microcontroller for its economic reason and multifunction. It is more compact, flexible, and cheap that are capable of doing more function than other predecessors that happened to cover more space, turned out costly with the ability to perform fewer functions.

Figure 4 shows flow chart of detecting the infrared signals of different buttons of the remote control. Firstly, we directly connected the Arduino UNO board with the infrared signal receiver to the computer. Placing the remote control towards the receiver and pressing the bottom that we want, then decimal signals were shown on the computer screen. We recorded them and they will be used in the setting actions and matched to different buttons.

Table 1 illustrates the infrared radiation signals with the decimal system. We decide 2 to control the forward movement of the easer; 8 for backward; 4 for turning left; 6 for turning right and 5 for stop. Figure 5 demonstrates the whole program of the remote control and eraser.

\subsection{Circuit Design}

The circuit connecting the battery and the electric motor is shown below in Figure 6. We use two 1.5 $\mathrm{V}$ batteries (Nanfu Batteries) to provide electric power. Batteries can replace charger wire and make the eraser more flexible. When switch 1 (the one that is on the top of the chalkboard eraser) closes, the electric motor connects to the rotary vane pump will be turned on and the pump the air in the chamber, causing negative pressure between the chalkboard eraser and the chalkboard. In this way, the eraser can stick to the chalkboard and being moved around. When we try to turn left, switch 3 will close (electric motor on the right) and when we try to turn right, Switch 2 will close (electric motor on the left). Switch 2 and 3 are controlled by Arduino. Details about the principle of turning will be illustrated in Section 2.5.

\subsection{Rotary Vane Pump}

We use a rotary vane pump in the chalkboard eraser. The structure of the pump is shown in Figure 7.

As the rotors being rotated by the electric motor, gas (or liquid) will be taken into the pump through the inlet (with blue arrows on the left) and exit the pump through the outlet (with red arrows on the right) by the push of vanes. There are various types of pumps as they are shown in Figure 8. Our eraser is light and does not require quite low pressure to generate sufficient friction between the eraser and the chalkboard. A small rotary vane pump can achieve such need and meanwhile is low-cost and easy to obtain. There will be one button on the top of the eraser that can be used to control the pump. 
Table 1. Infrared radiation signals with the decimal system.

\begin{tabular}{cc}
\hline BUTTON & SIGNAL \\
\hline 2 & 16718055 \\
4 & 16716015 \\
6 & 16734885 \\
8 & 16730805 \\
5 & 16726215 \\
\hline
\end{tabular}

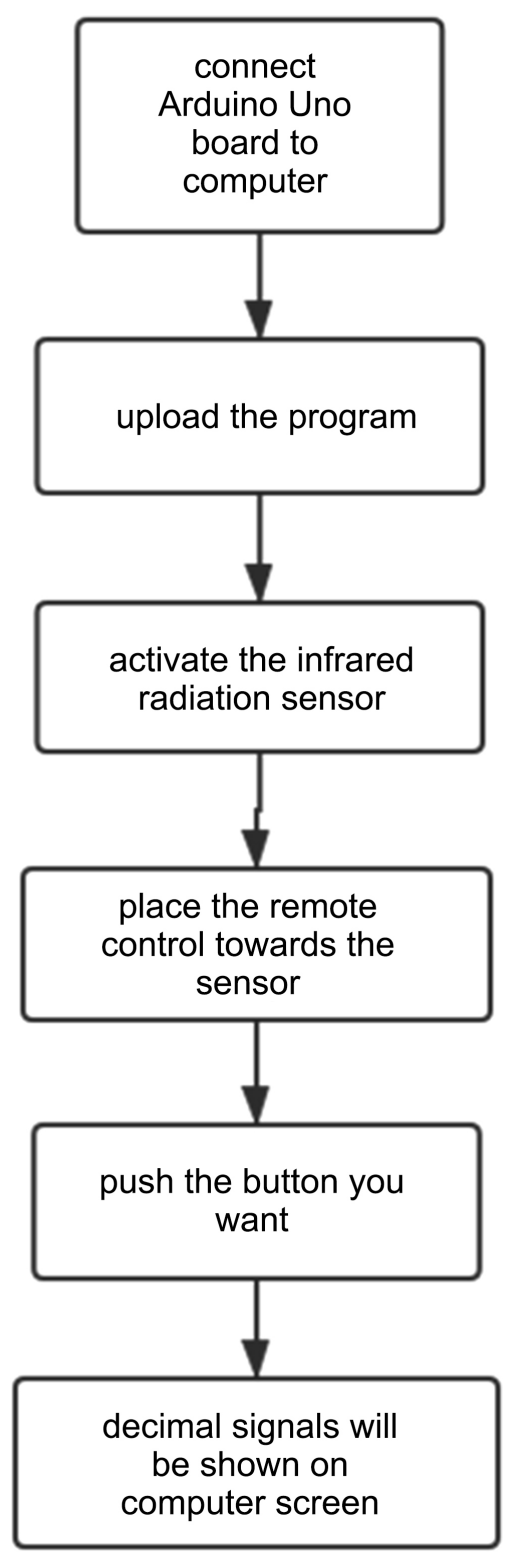

Figure 4. Flow chart of detecting the infrared signal of different buttons. 


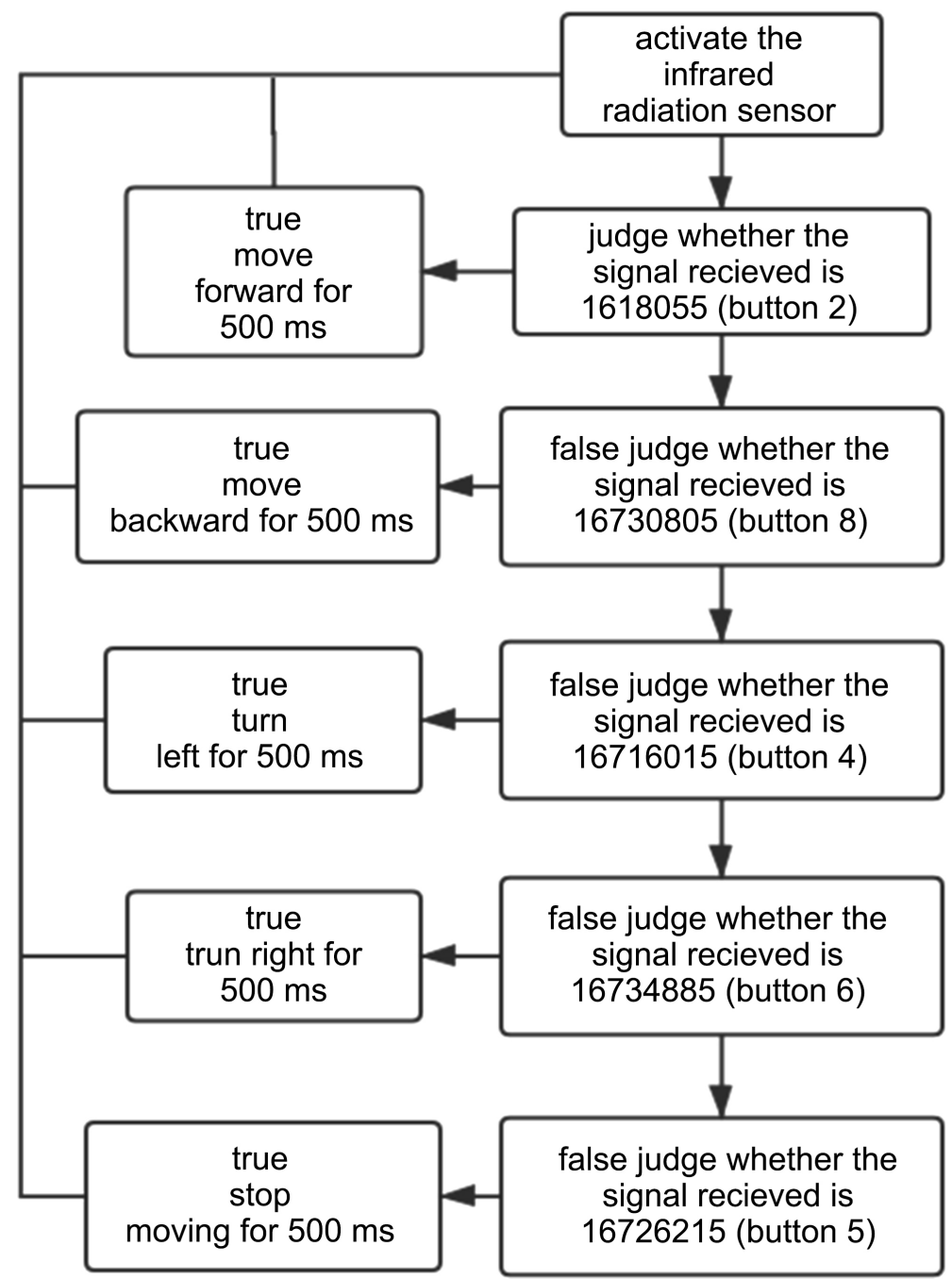

Figure 5. The flow chart of the remote-controlled system.

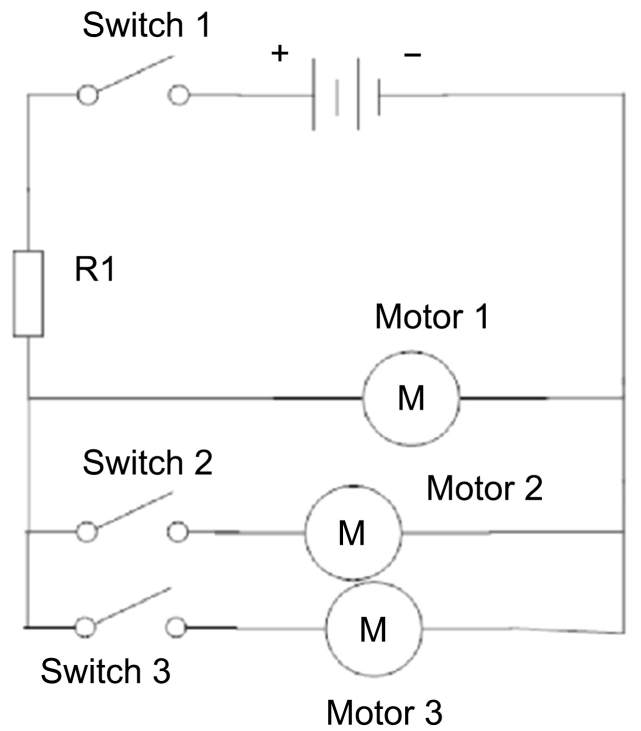

Figure 6. The circuit design of the remote-controlled eraser. 


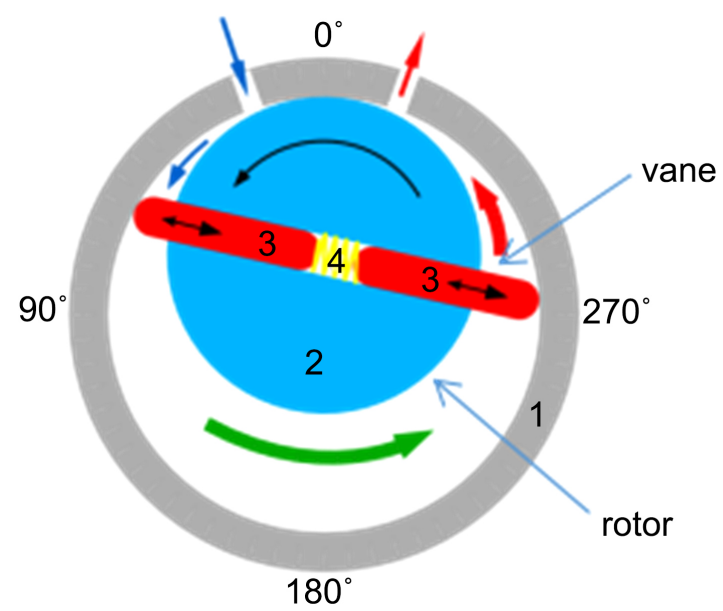

Figure 7. The working principle of a rotary vane pump from:

(https://www.cleanpng.com/png-rotary-vane-pump-vacuum-pump-compressor-hydraulic-813040/d ownload-png.html).

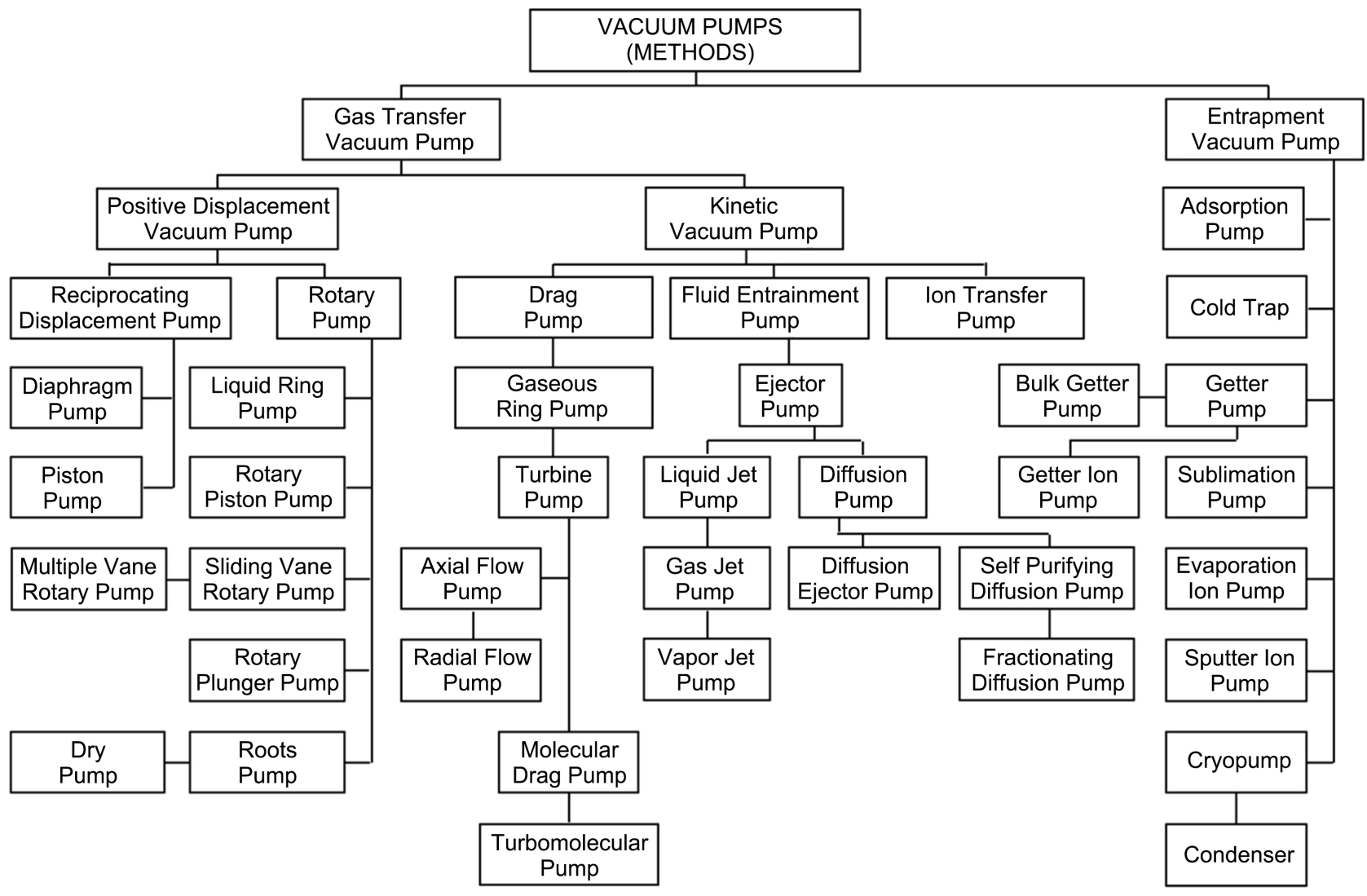

Figure 8. Different methods of vacuum pumps.

\subsection{Cleaning Materials}

The cleaning material commonly used in the chalkboard eraser has several drawbacks including the low efficiency in removing dust from the board especially when there are a large amount and the poor ability in collecting the dust after being removed from the board. To improve the performances in these areas, we choose microfiber as our cleaning material. Microfibers are tiny artificial fibers constructed po- 
lyester and polyamide. (structure of microfiber is shown in Figure 9) Microfiber products are made of fibers that are nearly $1 / 160$ the size of a human hair and therefore they are able to deep clean the pores of the microscopic surface of any materials (the working principle is shown in Figure 10) During the process of erasing on the board, microfibers will be positively charged and the dust will be negatively charged due to the transfer of electrons. This gives microfibers a stronger ability to collect dust than conventional materials (Figure 11).

\subsection{The Wheel System}

We use the differential wheel system to operate our chalkboard eraser on the chalkboard. The principle of the differential wheel system is shown in Figure 12.

The wheels are connected to electric motors that can be controlled by Arduino. When we try to turn left (press the left button on the controller), the left electric motor will not rotate the left wheel while the right one will go forward. The difference between velocities on two sides will rotate the eraser towards the left. The situation is similar when we try to turn right, the right wheel will not rotate while the left wheel will go forward. In this way, we can achieve turning around to any degree.

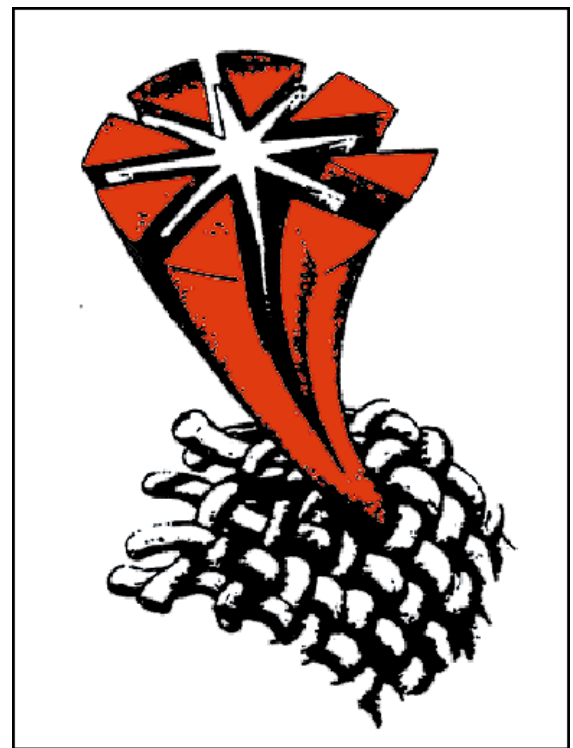

Figure 9. The structure of microfibers.

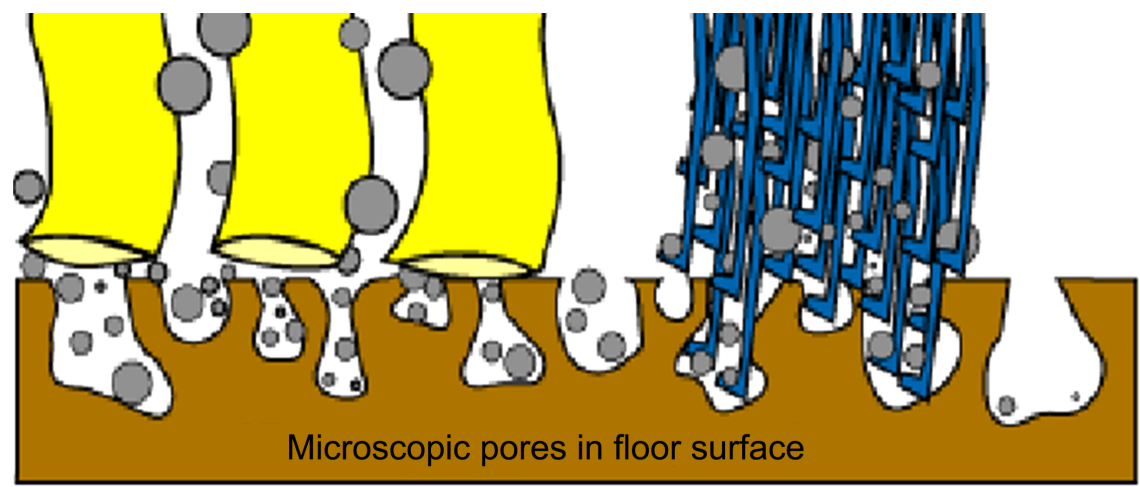

Microfibers physically go into microscopic pores

in surfaces to remove dirt, dust \& microdust.

Figure 10. The working principle of microfibers. 


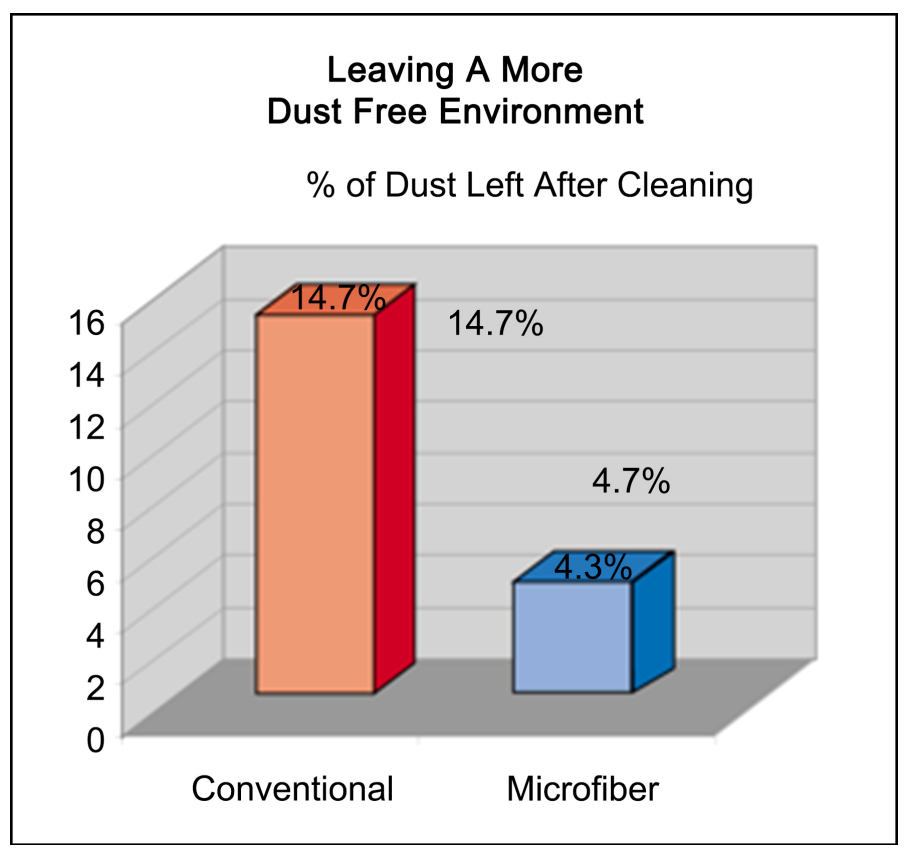

Figure 11. The comparison of the ability to collect the dust between conventional materials and microfibers of the working principle of microfibers.
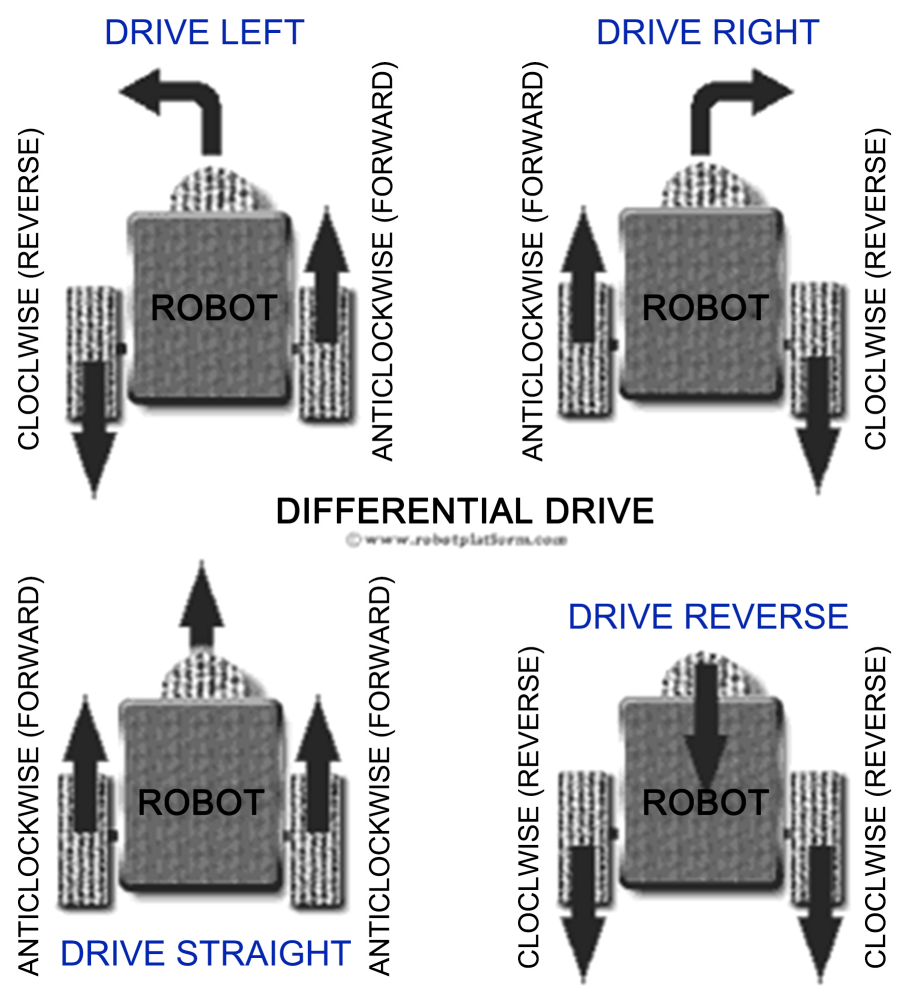

Figure 12. The working principle of a differential wheel system (higher rate of rotation at the right wheel will turn the vehicle to the left; higher rate of rotation at the left wheel will turn the vehicle to the right) from:

http://www.robotplatform.com/knowledge/Classification_of_Robots/wheel_c ontrol_theory.html. 


\section{RESULTS AND DISCUSSIONS}

Before you begin to format your paper, first write and save the content as a separate text file. Keep your text and graphic files separate until after the text has been formatted and styled. Do not use hard tabs, and limit use of hard returns to only one return at the end of a paragraph. Do not add any kind of pagination anywhere in the paper. Do not number text heads-the template will do that for you.

Finally, complete content and organizational editing before formatting. Please take note of the following items when proofreading spelling and grammar:

\subsection{Result}

The final version of our eraser has several improvements and newly added features compared with the traditional one. Firstly, this eraser can remove most of the dust that attaches to the blackboard to ensure its cleanliness and optimize both learning and teaching experiences. Secondly, dust produced by chalks are also can be automatically collected by this eraser, so it reduces the possibility of dust suspending in the air and taken in by teachers and students. Moreover, it is also equipped with a remote control system to erase the works on boards manually.

\subsection{Discussion}

The automatic chalkboard eraser we designed has a satisfying ability in decreasing the amount of powder produced during the teaching process and can be remote controlled. However, it still requires a person to control it. In "Automatic positioning and dust-free blackboard writing removal system" the author states that the Radio Frequency Identification (RFID) technology could help the eraser to locate the writing on the chalkboard. RFID technology is composed of RFID tags, Reader, and eraser. The RFID tag can be placed on the writing that needs to be erased and the Reader under Wireless Local Area Network will locate the position of the tag by scanning and send the signal to the eraser so that the eraser can clean the writing automatically. With the help of RFID technology, it is possible to design a chalkboard eraser that can clean the chalkboard without any control from people. Besides, the eraser we designed may not be suitable for intense teaching paces in classes as the ability to clean the dust sets a limit for the speed of moving around. If the eraser goes too fast, the ability to clean the dust will be weakened and vice versa. Therefore, it is important to find a balance between two factors, where the eraser can take in most of the dust produced while does not affect the pace of teaching. As technology develops, the microfiber in the eraser could also be replaced with better quality and functional materials to improve durability and ability to absorb the dust. In addition, the Arduino UNO board is used only for some basic functions, we can update it to other micro boards, e.g. Arduino Due, which have faster processing speed and behave more accurately.

\section{CONCLUSIONS}

To decrease the amount of the dust produced and inhaled by teachers and students during the use of chalks, we successfully designed a remote-controlled blackboard eraser that can be remote-controlled to clean the writing on the blackboard.

With a rotary vane pump we could adhere the eraser to the blackboard by taking out the air inside a sealed chamber between the eraser and the blackboard. With the Arduino UNO board, PIR sensor and the differential wheel system, we could write a program to the Aduino board and connect it to the PIR sensor so that it can recognize signals coming from different buttons on the controller. If we want to turn the eraser to the right, we can press the button on the controller that will instruct the Arduino board to turn the left side of the wheel to rotate faster than the right side one, which will turn the eraser to the right. The microfibers, a strong cleaning material, enable us to collect most of the dust produced while cleaning the blackboard due to its small diameters and outstanding strength.

In conclusion, we made the following achievements: 
- Use the rotary vane pump to create negative pressure between the eraser and the blackboard.

- Use an Arduino UNO board and a PIR sensor to achieve remote control.

- Use differential wheel system to enable the eraser to move around.

- Use microfibers to give the eraser an outstanding ability to collect dust.

\section{ACKNOWLEDGEMENTS}

The completion of our research project (from choosing the topics to complete the report) takes us a whole summer vacation. We want to thank Mr. Curt T. Reimann, our directing teacher, for giving constructive comments on the structure and grammar of our report and help us out when we met difficulties in building the model.

\section{CONFLICTS OF INTEREST}

The authors declare no conflicts of interest regarding the publication of this paper.

\section{REFERENCES}

1. Majumdar, D., Gajghate, D.G., Pipalatkar, P. and Chalapati Rao, C.V. (2011) Assessment of Airborne Fine Particulate Matter and Particle Size Distribution in Settled Chalk Dust during Writing and Dusting Exercises in a Classroom.

https://www.researchgate.net/publication/230640086_Assessment_of_Airborne_Fine_Particulate_Matter_and_ Particle_Size_Distribution_in_Settled_Chalk_Dust_during_Writing_and_Dusting_Exercises_in_a_Classroom

2. Ye, M., Cai, Z., Xin, B. and Wang, T. (2019) Optimization Design of New Type of Vacuum Eraser. Hubeinongjihua, No. 9, 73-74.

3. Jiang, W., Hou, Q. and Hou, Q. (2012) Design of Vacuuming Eraser. Theoretical Research, 8, 73, 76.

4. Frothingham, S. (2019) What's the Average Hand Size for Men, Women, and Children? https://www.healthline.com/health/average-hand-size

5. What Is Infrared? https://arvindunimap.com/infrared/

6. How Remote Control Work. https://electronics.howstuffworks.com/remote-controll.htm

7. Infrared Sensor-How It Works, Types, Applications, Advantage \& Disadvantage. https://electricalfundablog.com/infrared-sensor/ 